\title{
Implications of the cosmic ray spectrum from the second knee to the ankle region for the cosmic ray proton sources
}

\section{Eiji Kido* for the Telescope Array Collaboration ${ }^{\dagger}$}

Institute for Cosmic Ray Research, University of Tokyo, 5-1-5 Kashiwanoha, Chiba 277-8582, Japan

E-mail: ekidodicrr.u-tokyo.ac.jp

The Telescope Array and the Telescope Array Low Energy Extension experiment provides energy spectra of cosmic rays with energies above $10^{15.3} \mathrm{eV}$ using the same energy calibration techniques of fluorescence detectors. The calibration techniques enable the wide range of energy spectra to be combined with small systematic uncertainty. The energy spectra from below the second knee to the ankle are fitted with cosmic ray protons from a simple phenomenological extragalactic source model and with some galactic cosmic ray models. The energy spectra can be successfully fitted with some galactic cosmic ray models which show steep break at the second knee.

36th International Cosmic Ray Conference -ICRC2019-

July 24th - August 1st, 2019

Madison, WI, U.S.A.

\footnotetext{
${ }^{*}$ Speaker.

† for collaboration list see PoS(ICRC2019)1177
} 


\section{Introduction}

The Telescope Array (TA) experiment exhibited energy spectrum measured with the surface detector (SD) [四]. The TA experiment also showed energy spectra using hybrid events measured by both SD and fluorescence detector (FD) in [] $]$ and [B]. The energy spectrum obtained by the monocular analysis of FD was also published [四]. The Telescope Array Low Energy Extension (TALE) experiment showed energy spectra of cosmic rays with energies above $10^{15.3} \mathrm{eV}$ [5]. The energy scale of these energy spectra is determined calorimetrically by FDs. SD energies are rescaled by FD energies using SD FD hybrid events. All energy spectra are consistent with each other in their overlap energy region. This enables the wide energy range of the energy spectra above $10^{15.3} \mathrm{eV}$ until the highest energies to be evaluated with the common energy scale. This is one of the unique points of TA and TALE now.

In this energy range, the observed energy spectra show some breaks known as "knee", "second knee" and "ankle" and show suppression at the highest energies. The acceleration of galactic cosmic rays could reach their maximum energy at the knee and the second knee as one possibility. The transition from galactic cosmic rays to extragalactic cosmic rays was implicated in the ankle in the publications such as [6]. The ankle was explained as the dip feature of $\mathrm{e}^{+} \mathrm{e}^{-}$pair production of extragalactic cosmic ray protons on cosmic microwave background (CMB) photons in [प]. This model also explains the suppression at the highest energies because of pion production of cosmic ray protons on CMB photons. Pierre Auger observed the transition to heavier composition significantly from the ankle to the highest energies, so the energy spectrum above the ankle was interpreted with extragalactic source model separately from the component below the ankle [ [] ]. Both the ankle and the transition of composition are explained by introducing the interaction of cosmic ray nuclei with the photons around the sources without introducing transition between extragalactic sources in [Q] .

The second knee is observed at $10^{17.04} \mathrm{eV}$ by TALE FD and we assumed that the transition from the galactic cosmic rays to extragalactic cosmic rays occurs as the second knee in this paper. The extragalactic cosmic ray protons are also assumed to be dominant in the extragalactic component until around the ankle. Implications for the extragalactic cosmic ray proton sources are obtained with a simple phenomenological model and some galactic cosmic ray models under these assumptions.

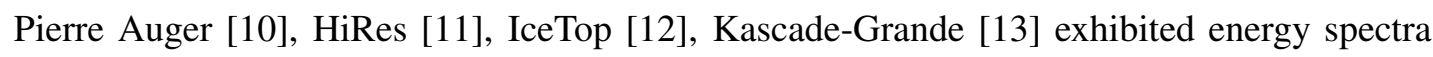
from the second knee to the highest energy region. Each experiment determined their energy scale independently and the systematic uncertainty is more than $14 \%$. As shown in Figure 1. in [[4]], their spectral shapes seem to be consistent except for the high or low energy end if the shift of the energy scale is applied. In this paper, the spectral feature above $10^{16.5} \mathrm{eV}$ below $10^{18.2} \mathrm{eV}$ of TA and TALE experiment is used, and the end of the energy spectrum is not used. The influence of the upper energy range $10^{18.2} \mathrm{eV}$ on the fit results is also checked.

The data set of TA and TALE used in this paper is shown in the section $\square$. The models and the procedure of the fit of the data is exhibited in the section [3. The fit results and the implications for the cosmic ray proton sources are shown in the section $\mathrm{A}$. The summary and conclusions are written in the section $\square$ and the discussion of the conclusions is shown in the section 6 . 


\section{Data set}

The full operation of the TA experiment continues for more than 10 years and TA SD energy spectrum was updated with 9 years data. The updated TA SD energy spectrum above $10^{18.2} \mathrm{eV}$ is used here because of the large statistics. TA SD can collect about 10 times larger number of events than TA FD because TA FD observes only at clear moon-less nights. To avoid using most of the same events, the energy spectrum of SD FD hybrid mode and the energy spectrum of TA FD monocular mode in the same energy region as TA SD are not used here. The energy spectrum of TA FD monocular mode between $10^{17.2} \mathrm{eV}$ and $10^{18.2} \mathrm{eV}$ obtained by the observation for 7 years is also used. TALE FD monocular mode energy spectrum between $10^{16.5} \mathrm{eV}$ and $10^{18.3} \mathrm{eV}$ obtained by about 3 years data is used because this energy range is wide enough to include the second knee

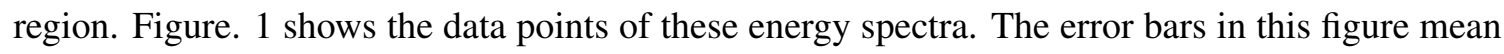
the statistical errors. The systematic uncertainty and statistical errors are combined in quadrature and shown as the color bands in the Figure. $\mathbb{~}$.

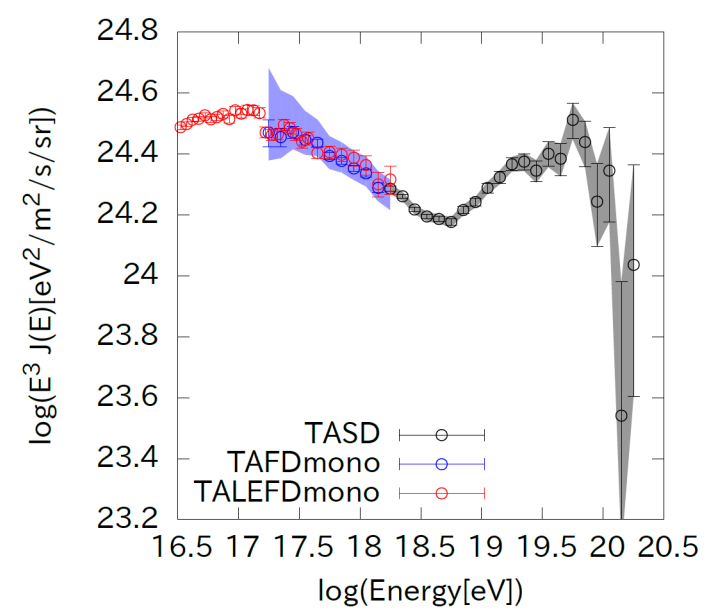

Figure 1: The black points: TA SD energy spectrum. The blue points: TA FD monocular energy spectrum. The red points: TALE FD monocular energy spectrum. The black and blue shade: uncertainty including systematic uncertainty of TA SD and TA FD monocular mode. The systematic uncertainty of the energy scale is considered separately from the uncertainty in this figure.

\section{Methodology}

The energy spectra shown in the previous section are fitted with an extragalactic cosmic ray proton source model and some galactic cosmic ray models. The model of the intensity $Q(E, z)$ of cosmic ray protons per unit volume from an extragalactic cosmic ray source at the redshift $z$ is

$$
Q(E, z)=\alpha E^{-p}(1+z)^{3+m}, E<E_{\max }, z<z_{\max }
$$

where $\alpha, p, m, z_{\max }$ and $E_{\max }$ are free parameters. If $m=0$, the number of sources per comoving unit volume does not change depending on $z$. The energy losses in the propagation of cos- 
mic ray protons with cosmic microwave background (CMB) and infrared (and optic) background (IRB) are calculated with an open source code TransportCR [16]. The calculation of the energy losses is checked with CRPropa [[1]]. The code implements several IRB models. Unless otherwise stated below we use the "best-fit" model in the reference [ए8] for IRB. The existing model calculations of the IRB spectral density can be different by factor of 2 at $z=0$ and moreover the uncertainty grows with redshift. The influence of the systematic difference of IRB models on the

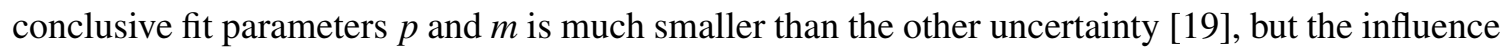
is large on the constraint of secondary photons as shown in the reference [[D]]. The constraint of the secondary neutrinos and photons from the experimental data is discussed in the section 42 . The propagation of cosmic rays can be different depending on the intergalactic magnetic field (IGMF). Current theoretical and observational constraints on the field strength $B$ of the IGMF summarized in the review [ [D] is $10^{-17} \mathrm{G}<B<10^{-9} \mathrm{G}$. The lower bound is updated to be $3 \times 10^{-16} \mathrm{G}<B$ for a coherent length $l_{c}>10 \mathrm{kpc}$ [D2]. The influence of the IGMF is not considered in this paper. The galactic cosmic ray model in [14] was used to fit the energy spectra.

The fit energy range is set to be between $10^{16.5} \mathrm{eV}$ and $10^{18.2} \mathrm{eV}$ as introduced in the section 四. Fit parameters are $\alpha, p, m$ and $z_{\max }$. The range of the fit parameter $z_{\max }$ is set to be between 0.6 and 5. The number evolution of many astrophysical sources approximately follows the power law of $(1+z)$ where the redshift is less than about 1 and the minimum value of the range 0.6 is taken.

The systematic uncertainty of each energy spectrum is included as the followings. The systematic uncertainty in [ㄷ] is used for TA SD energy spectrum and the statistic errors are combined in quadrature. The statistical errors and systematic uncertainty are also combined in quadrature for the energy spectrum of TA FD monocular analysis. Only the statistical errors are considered for the TALE FD monocular analysis because only the systematic uncertainty of the energy scale is shown in [[]]. Binned likelihood for Poisson distribution is maximized for TALE FD mono energy spectrum. Binned likelihood for Gaussian distribution is maximized for TA SD and TA FD mono energy spectrum to treat the uncertainty including the systematic uncertainty as Gaussian distribution. The log likelihoods of 3 energy spectra are combined by simply taking summation. 6 data points of the monocular analysis of TA FD and 29 data points of the monocular analysis of TALE FD are used for the fitting.

\section{Results}

The fit results of the experimental data are shown in this section. In the section 4. Ih, fitted results ignoring the magnetic field are shown. Practically $E_{\max }=10^{18.5} \mathrm{eV}$ is fixed in the section 4.D. The constraint from the observation of neutrinos and photons are shown in the section 42 .

\subsection{Fit results}

The energy spectra are fitted using 4 fit parameters as shown in the previous section [3. The best fit curves are plotted in Figure. $\square . z_{\max }$ determines the break of the low energy end of the extragalactic component and it is difficult to determine the detailed spectral shape of the low energy end of the extragalactic component from the data. Similar break can also be produced by the IGMF. 


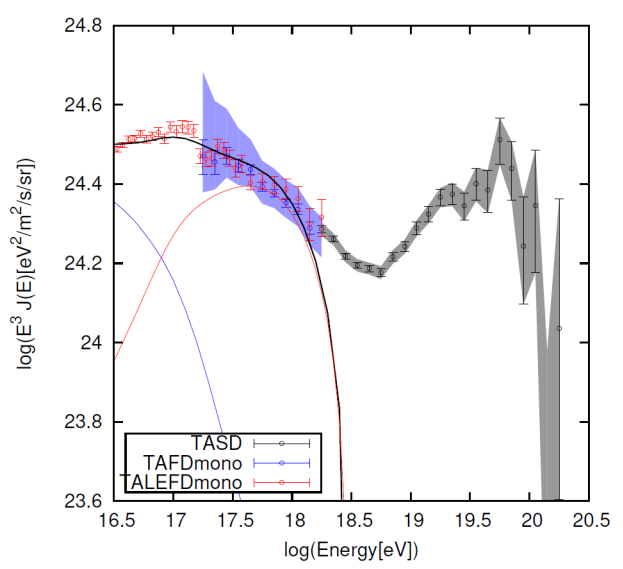

Figure 2: Same data points as Figure 1 are overlaid with the best fit model curves assuming $p=2.40, m=$ $2.7, z_{\max }=3.0$ and $E_{\max }=10^{18.5} \mathrm{eV}$. The black solid line is the best fit model curve. The red solid line and the blue solid line denote the extragalactic and galactic component, respectively.

\subsection{Secondary neutrinos and photons}

The electromagnetic cascades and secondary neutrinos from propagation cosmic ray protons are calculated with the calculation code in [ए]] and the cross check of the calculations was done with another code []]. The isotropic diffuse gamma ray background (IGRB) gives strong limit on secondary photons from cosmic ray protons. The IGRB from 50 months observation of Fermi LAT [23] is plotted in Figure. [3. 3 galactic foreground models were used in the calculation of IGRB. The galactic foreground model $\mathrm{B}$ gave highest estimate of IGRB and the least restrictive for cosmic ray protons. The upper limit defined in equation 13 in [20] above $50 \mathrm{GeV}$ which was obtained by the analysis in [24] is also shown in this figure. The observation of the IceCube experiment also provides strong constraint on secondary neutrinos from cosmic ray protons and the upper bound in this figure is from Figure. 10 in [25].

\section{Summary and Conclusions}

The energy spectra of TA SD, TA FD mono and TALE FD mono are now in public and their energy scales are calibrated in the same way. Simple phenomenological extragalactic proton sources and galactic cosmic ray model in [14] are assumed and attempted to fit the data with energies from $10^{16.5} \mathrm{eV}$ until $10^{18.2} \mathrm{eV}$. It is possible to fit the data successfully.

\section{Discussions and Future Prospects}

The results of the composition of cosmic ray nuclei will be provided by the TALE experiment. The agreement between the model and the observed composition will be considered. If gamma rays and neutrinos are observed in more detail at their high energy end in the future, this model 


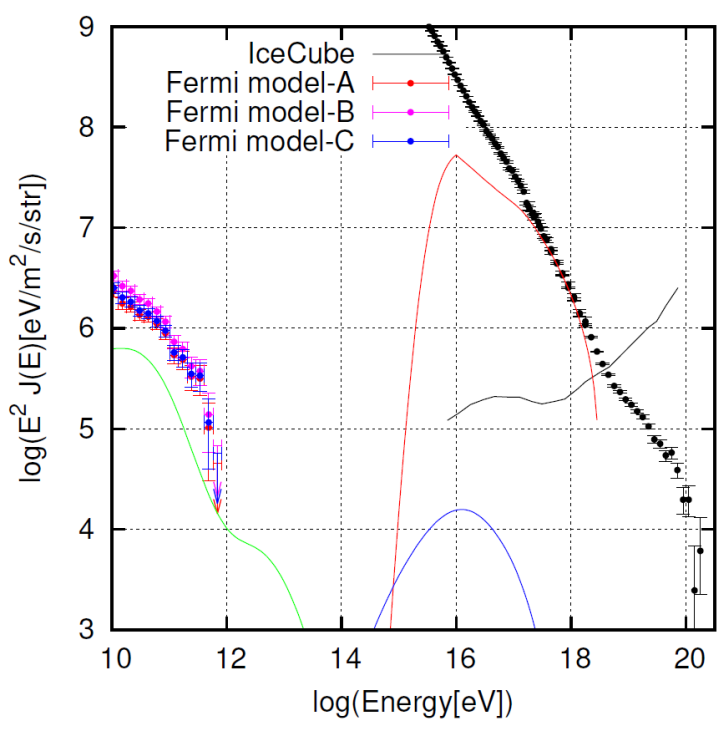

Figure 3: Red solid line denotes the best fit model curve shown in Figure 2. This model curve assumes $p=2.40, m=2.7, z_{\max }=3.0, E_{\max }=10^{18.5} \mathrm{eV}$ and $E_{\min }=10^{16} \mathrm{eV}$. The same data points as in Figure 2 are also plotted. Blue solid line and green solid line denote secondary neutrinos and secondary photons from cosmic ray protons, respectively. IGRB energy spectra observed by Fermi LAT for 3 galactic foreground models are also shown. The black solid line denotes the upper limit given by the observation of the IceCube experiment.

may be constrained more strongly. The implications of energy spectrum anisotropy with $E>10^{19.2}$ $\mathrm{eV}$ [26] and at the highest energies in [27] and [[28] were obtained by the TA experiment. Each significance of the anisotropy is estimated to be less than $4 \sigma$ confidence level, but if the anisotropy is true anisotropic energy spectrum will be fitted with more complex model. Especially the spectral feature at the highest energies will be studied by the TAx4 experiment [29] in detail in the next few years.

\section{References}

[1] T. Abu-Zayyad, R. Aida, M. Allen et al., The Cosmic-Ray Energy Spectrum Observed with the Surface Detector of the Telescope Array Experiment, ApJ., 768L (2013) 1A.

[2] T. Abu-Zayyad, R. Aida, M. Allen et al., Energy spectrum of ultra-high energy cosmic rays observed with the Telescope Array using a hybrid technique, Astropart. Phys., 61 (2015) 93.

[3] R. Abbasi, M. Abe, T. Abu-Zayyad et al., The hybrid energy spectrum of Telescope Array's Middle Drum Detector and surface array, Astropart. Phys., 68 (2015) 27.

[4] R. Abbasi, M. Abe et al., The energy spectrum of cosmic rays above $10^{17.2} \mathrm{eV}$ measured by the fluorescence detectors of the Telescope Array experiment in seven years, Astropart. Phys., 80 (2016) 131. 
[5] R. Abbasi, M. Abe et al., The Cosmic Ray Energy Spectrum between 2 PeV and 2 EeV Observed with the TALE Detector in Monocular Mode, ApJ., 865 (2018) 74A.

[6] D. J. Bird, S. C. Corbató, H. Y. Dai et al., The cosmic-ray energy spectrum observed by the Fly's Eye, ApJ., 424 (1994) 491.

[7] V. S. Berezinsky, A. Gazizov, \& S. Grigorieva, On astrophysical solution to ultrahigh energy cosmic rays, Phys. Rev. D, 74 (2006) 043005.

[8] A. Aab, P. Abreu, M. Aglietta et al., The Pierre Auger Observatory: Contributions to the 35th International Cosmic Ray Conference (ICRC 2017), [astro-ph. HE/1708 . 06592].

[9] M. Unger, G. R. Farrar, L. A. Achordoqui, Origin of the ankle in the ultra-high energy cosmic ray spectrum and of the extragalactic protons below it, Phys. Rev. D, 92 (2015) 123001.

[10] F. Fenu, The cosmic ray energy spectrum measured usingthe Pierre Auger Observatory, POS (ICRC2017) 486.

[11] R. Abbasi, T. Abu-Zayyad, M. Allen, et al., First Observation of the Greisen-Zatsepin-Kuzmin Suppression, Phys. Rev. Lett. 100 (2008) 101101.

[12] K. Rawlins, Cosmic ray spectrum and composition from three years of IceTop and IceCube, J. Phys. Conf. Ser. 718 (5) (2016) 052033.

[13] W. D. Apel et al., Kneelike Structure in the Spectrum of the Heavy Component of Cosmic Rays Observed with KASCADE-Grande, Phys. Rev. Lett. 107 (2011) 171104.

[14] T. Abu-Zayyad, D. Ivanov, et al., The Knee and the Second Knee of the Cosmic-Ray Energy Spectrum, [astro-ph.HE/1803.07052].

[15] D. Ivanov, PhD thesis, Rutgers, The State University of New Jersey, (2012).

[16] O. E. Kalashev and E. Kido, Simulations of ultra-high-energy cosmic rays propagation, JETP, 120, 5 (2015) 790.

[17] R. A. Batista, A. Dundovic, M. Erdmann, et al, CRPropa 3 - a Public Astrophysical Simulation Framework for Propagating Extraterrestrial Ultra-High Energy Particles, JCAP, 1605 (2016) 038.

[18] T. M. Kneiske et al., Implications of Cosmological Gamma-Ray Absorption II. Modification of gamma-ray spectra, $A \& A S, 413$ (2004) 807.

[19] E. Kido \& O. E. Kalashev, Interpretation of the energy spectrum observed with the Telescope Array surface detectors, in Proc. of ICRC2015 (Hague) (2015) 299.

[20] V. Berezinsky, A. Gazizov \& O. Kalashev, Cascade photons as test of protons in UHECR, Astropart. Phys., 84 (2016) 52.

[21] R. Durrer \& A. Neronov, Cosmological Magnetic Fields: Their Generation, Evolution and Observation, Astron. Astrophys. Rev. 21 (2013) 62.

[22] M. Ackermann, M. Ajello, L. Baldini, et al., The Search for Spatial Extension in High-latitude Sources Detected by the Fermi Large Area Telescope, ApJS, 237 (2018) 32.

[23] M. Ackermann, M. Ajello, A. Albert et al., The spectrum of isotropic diffuse gamma-ray emission between $100 \mathrm{MeV}$ and $820 \mathrm{GeV}, \mathrm{ApJ} ., 799$ (2015) 86.

[24] M. Di Mauro, The origin of the Fermi-LAT gamma-ray background, [astro-ph.HE/1601.04323]. 
[25] M. G. Aartsen, M. Ackermann, J. Adams et al., Differential limit on the extremely-high-energy cosmic neutrino flux in the presence of astrophysical background from nine years of IceCube data, Phys. Rev. D 98 (2018) 062003.

[26] R. U. Abbasi, M. Abe, T. Abu-Zayyad et al., Evidence of Intermediate-scale Energy Spectrum Anisotropy of Cosmic Rays $E \geq 10^{19.2} \mathrm{eV}$ with the Telescope Array Surface Detector, A\&A $\mathbf{8 6 2}$ (2018) 91.

[27] R. U. Abbasi, M. Abe, T. Abu-Zayyad et al., Search for Anisotropy in the Ultra High Energy Cosmic Ray Spectrum using the Telescope Array Surface Detector, [astro-ph. HE/1707.04967].

[28] R. U. Abbasi, M. Abe, T. Abu-Zayyad et al., Evidence for Declination Dependence of Ultrahigh Energy Cosmic Ray Spectrum in the Northern Hemisphere, [ast ro-ph. HE / 1801 . 07820].

[29] E. Kido, The TAx4 experiment, 